Manuscript \#: BM-D-16-00495R1, Rev. 2, Original

Original Article:

\title{
Lumped-Parameter Electromyogram-Driven Musculoskeletal Hand Model: A Potential Platform For Real-Time Prosthesis Control
} \author{
University, Raleigh, NC, 27695 \\ Corresponding Author \\ Name: $\quad$ Dustin L. Crouch, PhD \\ Address: $\quad$ North Carolina State University \\ 1407 Engineering Building 3 \\ 911 Oval Drive \\ Raleigh, NC 27695 \\ Email: $\quad$ dlcrouch@ncsu.edu \\ Phone: $\quad$ (919) 515-4412 \\ Fax: $\quad$ (919) $515-7760$
}

Dustin L. Crouch, $\mathrm{PhD}^{1}$, He Huang, $\mathrm{PhD}^{1}$

1. UNC-NC State Joint Department of Biomedical Engineering, North Carolina State

Keywords: wrist, simulation, amputation, parameter, optimization

Word Count: 3498 


\section{Abstract}

Simple, lumped-parameter musculoskeletal models may be more adaptable and practical for clinical real-time control applications, such as prosthesis control. In this study, we determined whether a lumped-parameter, EMG-driven musculoskeletal model with four muscles could predict wrist and metacarpophalangeal (MCP) joint flexion/extension. Forearm EMG signals and joint kinematics were collected simultaneously from 5 able-bodied (AB) subjects. For one subject with unilateral transradial amputation (TRA), joint kinematics were collected from the sound arm during bilateral mirrored motion. Twenty-two model parameters were optimized such that joint kinematics predicted by EMG-driven forward dynamic simulation closely matched measured kinematics. Cross validation was employed to evaluate the model kinematic predictions using Pearson's correlation coefficient ( $r$ ). Model predictions of joint angles were highly to very highly positively correlated with measured values at the wrist $A B$ mean $r=0.94$, TRA $r=0.92$ ) and MCP (AB mean $r=0.88$, TRA $r=0.93$ ) joints during singlejoint wrist and MCP movements, respectively. In simultaneous multi-joint movement, the prediction accuracy for TRA at the MCP joint decreased $(r=0.56)$, while $r$-values derived from $A B$ subjects and TRA wrist motion were still above 0.75 . Though parameters were optimized to match experimental sub-maximal kinematics, passive and maximum isometric joint moments predicted by the model were comparable to reported experimental measures. Our results showed the promise of lumped-parameter musculoskeletal model for hand/wrist kinematic estimation. Therefore, the model might 
be useful for EMG control of powered upper limb prostheses, but more work is needed to demonstrate its online performance.

\section{Introduction}

Computational musculoskeletal models have been used extensively to investigate healthy and impaired human movement (Higginson et al., 2006; Zajac et al., 2002) and simulate surgery and rehabilitation (Delp et al., 1990; Saul et al., 2003; Shelburne and Pandy, 1998), among other applications. Forward dynamic simulation, when applied to musculoskeletal models, can generate joint kinematic predictions from input electromyogram (EMG) signals. Forward dynamic simulation has primarily been used off-line, for example, to estimate muscular biomechanical contributions to movement (Neptune et al., 2001) and joint loading (Manal and Buchanan, 2013). When executed in real-time, forward dynamic simulation could generate motion predictions for external devices, such as powered prostheses (Eilenberg et al., 2010) .

Many upper limb musculoskeletal models implemented for real-time EMG-driven forward dynamic simulation include numerous musculoskeletal elements to accurately represent anatomy and generate physiologic predictions. Model complexity has ranged from 5 muscles and 1 degree of freedom (DOF) to predict isometric elbow joint moments (Manal et al., 2002), to 138 muscles and 11 DOFs to investigate the complex motions of the arm and shoulder girdle (Chadwick et al., 2009). Unfortunately, it may be impractical to adapt anatomically-representative models for real-time clinical control applications, as an overwhelming number of parameters would need to be customized. Adjusting models parameters for amputees is even more challenging since (1) there is 
no intact musculoskeletal structure from which to measure parameters directly, and (2) the perceived biomechanical actions associated with EMG signals are not observable and may be altered from that of a healthy, intact limb due to cortical reorganization (Ramachandran and Hirstein, 1998). Measuring EMG for several muscles, especially deep muscles, may also be clinically challenging and subject to crosstalk. Finally, forward dynamic simulation of models with several musculoskeletal elements can be computationally intensive.

As a counter to complex models, lumped-parameter models that combine the action of several muscles into fewer muscle elements may be more clinically practical (Eilenberg et al., 2010; Lehman and Calhoun, 1990; Messier et al., 2011). Modeling fewer muscle elements reduces (1) the number of parameters that must be adjusted for each subject, (2) the number of input EMG signals, and (3) the computational burden of forward dynamic simulation. To streamline the development of subject-specific models, researchers have used numerical optimization to adjust existing or define novel parameters to minimize error between measured and predicted joint moments given input EMG signals (Lehman and Calhoun, 1990; Lloyd and Buchanan, 1996; Shin et al., 2009). However, methods based on joint moments cannot be applied for amputees since the moments in the missing joints are indeterminable. Though both motion and force mirroring have been used as an indicator of amputee movement intent (Kamavuako et al., 2012; Muceli and Farina, 2012), force may not be truly mirrored since amputees cannot actively resist external loads on their amputated side. Therefore, 
we propose to compute a novel set of musculoskeletal parameters for a lumpedparameter model to match measured joint kinematics.

The objective of our pilot study was to develop and preliminarily test a lumpedparameter, 2 DOF model of the hand. Specifically, we wanted to (1) demonstrate that the model could reasonably predict joint kinematics during single-joint and simultaneous two-joint movements for able-bodied subjects and a transradial amputee; (2) compare the active and passive joint moment-generating capacity of subjects' models to that reported for healthy, intact limbs; and (3) evaluate the performance and repeatability of numerical optimization for computing model parameters. Our findings may promote the development and translation of real-time musculoskeletal model-based forward dynamic simulation for multifunctional upper limb prosthesis control.

\section{Methods}

Experiments and Data Collection

Experiments were approved by the institutional review board. Five able-bodied (AB1-AB5) subjects (3 males, 2 females, age range 23-31 years) and 1 subject (TRA) with transradial amputation (male, age 42) provided informed consent to participate. Subject TRA sustained a right traumatic amputation approximately 2 years before participating, and regularly used a body-powered prosthesis.

In one session, subjects performed 5 different types of movement in a static upper limb posture with the arm and forearm in neutral posture and elbow flexed to $90^{\circ}$ : (1) wrist flexion/extension only, variable speed; (2) MCP flexion/extension only, variable 
speed; (3) simultaneous wrist and MCP flexion/extension, variable speed; (4) wrist flexion/extension only, fixed speed; and (5) MCP flexion/extension only, fixed speed. During variable speed movements, subjects moved in self-selected speeds and directions. In fixed speed trials, subjects alternated between maximum extension, relaxed, and maximum flexion joint postures at a regulated tempo $(0.25 \mathrm{~Hz})$. Ablebodied subjects performed the movements with the dominant arm, while subject TRA mirrored movements bilaterally. Each movement type was tested in two trials for at least 30 seconds; each subject performed a total of 10 trials $(5$ movements $\times 2$ trials/movement). Subjects rested between trials.

During trials, EMG and kinematic data were collected synchronously from ablebodied subjects' dominant limb; for subject TRA, EMG and kinematics were measured from the residual and sound limb, respectively. Four bipolar surface EMG electrodes (Biometrics, Newport, UK) were placed over muscles/muscle groups (Figure 1) identified by anatomical reference and palpation during experimenter-directed movements, and confirmed by visualizing EMG. The selected muscles generate wrist and metacarpophalangeal (MCP) flexion and extension joint moments in intact limbs based on their musculoskeletal geometry (Perotto, 2005). EMG data were sampled at $960 \mathrm{~Hz}$, high-pass filtered at $40 \mathrm{~Hz}$, rectified, enveloped, and low-pass filtered at $6 \mathrm{~Hz}$ using a $4^{\text {th }}$ order Butterworth zero-phase filter, similar to previous methods (Lloyd and Besier, 2003). EMG were then normalized by respective maximum (post-processed) EMG signal values recorded during maximum voluntary contractions. 
Given the short length of subject TRA's residual limb, EMG associated with wrist flexion appeared in electrodes targeting both wrist and MCP flexion, as flexor digitorum (MPC flexion) is deep to flexor carpi ulnaris (wrist flexion) in the proximal forearm. Therefore, similar to a previous method (Reddy and Gupta, 2007), we linearly transformed MCP flexor EMG $\left(E M G_{M C P \text { flex }}\right)$ to reduce EMG associated with wrist flexion, based on the approximate proportion of wrist flexor EMG ( $\left.E M G_{\text {wrist flex }}\right)$ appearing in $E M G_{M C P \text { flex }}$ during isolated wrist flexion movements (Eq.1):

$$
E M G_{M C P \text { flex }}^{*}=E M G_{M C P \text { flex }}-0.75 E M G_{\text {wrist flex }} \quad \text { Eq. } 1
$$

Reflective markers were placed on 9 anatomical locations to track distal limb motion (Figure 1); thumb motion was not recorded or included in the model. Threedimensional marker positions were recorded at $120 \mathrm{~Hz}$ using an infrared motion capture system (Vicon Motion Systems Ltd., UK), and filtered at $6 \mathrm{~Hz}$ using a $4^{\text {th }}$ order Butterworth filter. Wrist and MCP joint angles were computed from filtered marker data using a musculoskeletal model (Holzbaur et al., 2005a) in OpenSim (Delp et al., 2007) that was modified to include the $2^{\text {nd }}$ through $5^{\text {th }}$ MCP joints.

\section{Dynamic Hand Model}

We defined a planar link-segment dynamic model (dynamic properties described in Supplementary Data) with two degrees of freedom (DOFs) - wrist and MCP flexion/extension - that was encoded in MATLAB (MathWorks, Inc., Natick, MA). Four muscles, one for each EMG source, were represented as Hill-type actuators with a contractile element (CE) and parallel elastic element (PEE) (Winters, 1990). A series 
elastic element, commonly included in Hill-type models to represent tendon, was not included. The force output of the contractile element, $F^{C E}$, was a function of its length $\left(L^{C E}\right)$, shortening velocity $\left(v^{C E}\right)$, and state of activation (a) (Eq.2).

$$
F^{C E}=f\left(L^{C E}\right) f\left(v^{C E}\right) a
$$

Where $f\left(L^{C E}\right)$ is the active CE force (Eq.3) and $f\left(v^{C E}\right)$ is a hyperbolic scaling function that reduces $F^{C E}$ as $v^{C E}$ increases (Eq.4).

$$
\begin{array}{ll}
f\left(L_{C E}\right)=F_{0}^{C E}\left(1-\frac{\left(L^{C E}-L_{0}^{C E}\right)^{2}}{W^{2}\left(L_{0}^{C E}\right)^{2}}\right) & f\left(L^{C E}\right)>0.01 \\
f\left(v_{C E}\right)=\frac{v_{\max }^{C E}-v^{C E}}{v_{\text {max }}^{C E}+\left(\frac{v^{C E}}{c}\right)} & 0<f\left(v^{C E}\right)<1
\end{array}
$$

In Eq.3, $L_{0}^{C E}$ is the optimal CE length, and $w$, which defined the range over which the CE could produce force as a fraction of $L_{0}^{C E}$, was set at 0.5 (Buchanan et al., 2004). In Eq.4 the maximum CE shortening velocity $\left(v_{\max }^{C E}\right)$ was set to $10 \frac{L_{0}^{C E}}{\mathrm{~s}}$, and the hyperbolic shape factor $(c)$ was set to 0.25 (Winters, 1990; Zajac, 1989). The parallel elastic element generated force $\left(F^{P E E}\right)$ when its length exceeded $L_{0}^{C E}$ (Eq.5).

$$
F^{P E E}=K^{P E E}\left(L^{C E}-L_{0}^{C E}\right)^{2} \quad L^{C E}>L_{0}^{C E}
$$

Muscle activation states, ranging between 0 (inactive) and 1 (fully activated), were computed from EMG, accounting for the electromechanical delay between neural excitation, represented by EMG, and muscle force production (Lloyd and Besier, 2003). Net joint moments $\left(M_{j}\right)$ at joint $j$ were computed as the product of muscle force and 
moment arm (ma) for each muscle $n$, summed across all $k$ muscles ( $k=4$ in our model) (Eq. 6).

$$
M_{j}=\sum_{n=1}^{k} F_{n}^{C E} \times m a(n)_{j} \quad \text { Eq. } 6
$$

ma was assumed constant with respect to joint angle. Joint moments were applied during a forward dynamic simulation. Joint kinematics were computed by numerically integrating the equations of motion over $1 / 120$ second time intervals using a $4^{\text {th }}$ order Runge-Kutta method (Chadwick et al., 2009).

\section{Numerical Optimization}

Twenty-two musculoskeletal parameters, constrained to approximate physiologic ranges (Holzbaur et al., 2005b), were computed by constrained global numerical optimization. Six parameters were computed for each muscle (Table 1), except, for wrist flexor and extensor muscles that only crossed the wrist and had no moment arm at the MCP joint, $m a_{M C P}$ was set to zero. These parameters were chosen because they strongly influence the force- and moment-generating behavior of muscle.

For each subject, data from 5 of the 10 trials, one selected arbitrarily from each of the five movement types, were used for optimization. In each optimization loop, muscle activations were control inputs during a forward dynamic simulation. Since the range of motion of the wrist is greater than that of the MCP joint (Norkin and White, 2009), musculoskeletal parameters were optimized in order to minimize the weighted (MCP weight $=2 \times$ wrist weight) sum of squared differences between measured and predicted kinematics. Parameters were optimized for each individual subject using 
MATLAB's GlobalSearch function. For comparison, we optimized parameters for a randomly-selected able-bodied subject by simulated annealing (SA), a widely used technique for solving complex, non-linear biomechanical optimization problems (Higginson et al., 2005). Optimization settings are shown in Table 2.

\section{Data Analysis}

We estimated how well the model predicted subjects' wrist and MCP joint angles using a cross validation approach. We computed Pearson's correlation coefficient $(r)$ between measured and predicted angles over a contiguous 25 -second time window for variable speed trials not included in the optimization procedure. We also reported the strength of correlation (e.g. high, very high) based on a previously established scale (Hinkle et al., 2003). For subject TRA, model-predicted kinematics were compared to measured sound limb kinematics.

As further validation, we compared the passive and maximum isometric (passive + active) joint moments that subjects' models could generate (scaled to account for higher hand mass) with reported experimental values (Delp et al., 1996; Knutson et al., 2000; Mathiowetz et al., 1985) using a Welch's t-test for independent samples. Passive and maximum isometric wrist joint moments were computed with the MCP joint in neutral posture. Passive MCP joint moments were computed with the wrist in neutral posture. Maximum isometric MCP flexion moments were computed with the wrist in neutral posture and MCP in $45^{\circ}$ flexion to approximate subjects' posture during grip strength tests (Mathiowetz et al., 1985). 
Finally, we compared optimization results from GlobalSearch and SA algorithms. To assess optimization consistency, we executed 10 optimization routines with both GlobalSearch and SA algorithms for the aforementioned randomly-selected able-bodied subject. A paired Student's t-test was performed to compare prediction accuracy between GlobalSearch and SA (as measured by Pearson's correlation coefficient). Statistical differences were considered significant for $p<0.05$.

\section{Results}

Models with GlobalSearch-computed parameters (Table 3) reasonably predicted able-bodied subjects' wrist and MCP joint kinematics, even during simultaneous wristMCP joint movement (Figure 2 and Supplementary Data). For subject TRA, kinematic predictions driven by EMG measured from the residual limb matched the relative magnitude and direction of joint movement measured from the sound limb (Figure 3), though not as well as for able-bodied subjects. Offline, the model could execute an EMG-driven forward dynamic simulation of a 20-second trial in approximately 1.5 seconds, demonstrating that the model permits simulation speeds required for real-time control.

The model's joint angle predictions showed high (0.7-0.9) to very high $(>0.9)$ positive correlation ( $\mathrm{p}<0.001$ for all correlations) with measured joint angles across nearly all trials (Figure 4). Across able-bodied subjects, mean correlation coefficient ( $r$ ) values during wrist-only and MCP-only movements at those joints were $0.94(\mathrm{SD}=0.02)$ and $0.88(S D=0.02)$, respectively. Similarly, correlations for subject TRA were very high at both the wrist $(r=0.92)$ and MCP $(r=0.93)$ joints when they were moved 
independently. During simultaneous wrist-MCP movement, mean correlations across able-bodied subjects remained high at both the wrist (mean $r=0.89, S D=0.04$ ) and $\mathrm{MCP}$ (mean $r=0.75, \mathrm{SD}=0.06$ ) joints. For the same movement, subject TRA's predicted and measured kinematics were very highly correlated at the wrist $(r=0.93)$, but moderately correlated at the MCP joint $(r=0.56)$.

The model's force-generating behavior was generally consistent with that of healthy adults. Model-generated absolute passive moments were slightly, but not significantly, higher at the extremes of joint range of motion compared to experimental measurements; however, as in the biological limb, the joints were statically stable since passive forces tended to return joints to neutral posture (Figure 5). Likewise, the maximum isometric joint moments generated by the model were not significantly different from physiologic values (Figure 6).

For randomly-selected subject $A B 4$, ten repeated GlobalSearch optimization routines yielded a model with very consistent predictive capability. The mean and standard deviation of correlation coefficient between measured and predicted joint angles were $0.92(\mathrm{SD}=0.004)$ and $0.71(\mathrm{SD}=0.05)$ at the wrist and $\mathrm{MCP}$ joints, respectively (Figure 7). Variation in correlation coefficient was greater when parameters were computed by simulated annealing (mean $r=0.93, S D=0.006$ and mean $r=0.52$, $\mathrm{SD}=0.13$ at the wrist and MCP joints, respectively). Correlation coefficient at the wrist was only 0.01 greater for SA $(p<0.001)$, while correlation coefficient at the MCP joint was 0.19 lower for $S A(p<0.001)$. Both GlobalSearch and simulated annealing took 
approximately 20 hours to execute on a desktop computer (Dell Precision T5810, 3.70 GHz processor, 32 GB RAM).

\section{Discussion}

We demonstrated that a simple, lumped-parameter, EMG-driven musculoskeletal model of the wrist and hand could reasonably predict both single-joint and multi-joint wrist and MCP movements for both able-bodied and amputee subjects. As further validation, we found that the passive and maximum isometric joint moment-generating capacity of the model approximated that of healthy adults. Model predictions of submaximal kinematics were more accurate than passive and maximal active kinetic predictions, which was not surprising since the model parameters were computing using experimental sub-maximal kinematic data. However, it was notable that the model mimicked the passive static stability and approximate maximum isometric strength of the human hand, though it had substantially fewer muscles than the biological limb.

For clinical applications, an optimization approach may be better suited for defining model parameters than conventional methods based on direct anatomical measurements. Common sources of anatomical data include musculoskeletal specimens (Lieber et al., 1992; Murray et al., 1995), medical imaging (Holzbaur et al., 2007), or functional strength tests (Lehman and Calhoun, 1990). Such sources may be inappropriate for defining parameters in a lumped-parameter model whose muscles have no direct anatomical analog, and for amputees whose muscles no longer span the 
missing limb. However, if prediction accuracy is not compromised, defining values for some model parameters, such as moment arm, using experimental data may further simplify our modeling approach.

Despite searching over a relatively large set of 22 parameters, Matlab's GlobalSearch optimization algorithm, a gradient-based method, yielded better predictions than simulated annealing (SA), a stochastic method. However, other algorithms may search more efficiently and extensively over the parameter space. For instance, simulated annealing parallelized across multiple processors can test several points simultaneously (Higginson et al., 2005). Simplifying the optimization problem by reducing the number of optimized parameters, shortening the simulation in each iteration, obtaining prior knowledge, or further constraining parameter values may also improve efficiency. Sensitivity analyses are needed to identify appropriate simplifications that do not diminish optimization performance or prediction accuracy.

Several amputees, including our subject TRA, experience phantom limb sensations with voluntary muscle contraction (Ramachandran and Hirstein, 1998). This suggest that amputees retain an internal representation of their missing limb that may allow them to generate functionally-relevant motor commands after amputation (Mercier et al., 2006; Wolpert and Flanagan, 2001). That motor commands retain functional meaning is further evidenced in the ability of machine learning algorithms to distinguish among several different intended motions from multi-channel EMG (Li et al., 2010; Zhang and Huang, 2015). However, few have investigated the implicit, perceived biomechanical actions of individual residual muscles following amputation. That we 
could predict independent flexion and extension motion of the wrist and MCP joints for subject TRA suggests that amputees' residual muscles may retain unique functional roles that can be coordinated across several muscles to produce intentional multi-joint movements. By capturing the perceived biomechanical structure of an amputees missing limb, our model could help researchers further investigate these implicit functional roles to improve myoelectric prosthesis control.

MCP joint angle prediction accuracy (as quantified by Pearson's correlation coefficient) was lower across subjects during multi-joint movements than during singlejoint movements, especially for subject TRA. This may have been due to greater EMG crosstalk, a known limitation of surface electromyography, during MCP movement when the wrist was moved simultaneously. Additionally, EMG electrodes may have been placed imprecisely. Potential approaches and tools to acquire localized EMG signals include intramuscular EMG recording (Riek et al., 2000; Weir et al., 2009), spatial filters applied to high-density recordings (Huang et al., 2009), or medical imaging (Boon et al., 2011). Muscle loss, reduced somatosensory feedback, and motor reorganization may also influence the quality and accessibility of EMG recordings from amputees. These challenges have been addressed by emerging technologies, such as targeted nerve reinnervation to reaccess neuromuscular control signals to the missing limbs (Kuiken et al., 2009) and nerve stimulation to restore natural sensation (Tan et al., 2014). Our model may benefit from these technologies and, in turn, accurately estimate amputee movement intent in a novel way to advance multifunctional prosthesis control and 
restore functional motor coordination patterns in amputees. Combining our model with these advanced neural technologies will be interesting future work.

\section{Limitations}

The study included relatively few subjects, including only one individual with transradial amputation, so it is unclear whether the model performance would be consistent across the clinical population. With further development, we plan to evaluate the model's offline and real-time performance for more subjects to more broadly characterize its clinical viability as a prosthesis control platform.

Several simplifying assumptions, some of which are discussed below, were made to design a model that would be practical for prosthesis control, as a more complex model may increase computation time and the number of parameter values to compute or estimate.

1. We did not include tendons, which store elastic energy and transmit force from muscles in the forearm to the joints of the wrist and hand, allowing much of the muscle mass contributing to hand function to be located proximally. For a given muscle-tendon length, tendon reduces the excursion of the CE so that, through a given range of joint rotation, the CE operates over a smaller portion of the forcelength curve. This effect of tendon on muscle-tendon mechanics could be somewhat replicated in our model by a CE only with a longer optimal length. Accounting for musculotendon dynamics would require additional computational resources, making real-time prosthesis control more challenging to achieve. 
2. Muscle moment arms were assumed constant with respect to joint angle. Some muscles (e.g. flexor carpi radialis, extensor digitorum) that cross the wrist and MCP joints have relatively constant moment arms, while others (e.g. extensor carpi radialis longus, flexor digitorum superficialis) do not (An et al., 1983; Gonzalez et al., 1997).

3. Only four muscles were included in the model, though there are over 40 muscles that may contribute to wrist and hand movement (Jacobson et al., 1992; Keir et al., 1996).

4. The model represented wrist and finger motion, but did not include a thumb, which is critical for grasping objects. Myoelectric prostheses typically do not enable explicit control of thumb motion, but rather include it implicitly in the design and functionality. For instance, a prosthetic thumb mechanism opposing the fingers can be fixed or commanded to flex when the finger mechanism flexes (i.e. finger flexion in our model) to enable grasp.

Our simplification of the hand likely contributed to kinematic prediction errors. Whether the model enables effective real-time control despite prediction errors will be the focus of future studies.

\section{Conclusion}

In conclusion, our musculoskeletal model yielded reasonably accurate predictions of single-joint and simultaneous multi-joint movements. The model's simplified structure and optimization-derived parameters make it more practical to adapt to clinical populations while still mimicking the dynamic behavior of the biological limb. 
Though primarily intended as a clinical tool for real-time prosthesis control, the model may be useful for investigating neuromuscular control and adaptation in healthy and impaired individuals.

\section{Acknowledgements}

This work was sponsored by the Defense Advanced Research Projects Agency (DARPA) Biological Technologies Office (BTO) Hand Proprioception and Touch Interfaces (HAPTIX) program under the auspices of Dr. Doug Weber through the DARPA Contracts Management Office Grant/Contract No. N66001-16-2-4052.This project was also supported by NSF \#1527202, DHHS/NIDILRR \#90IF0064, and DOD \#OR140147 \& \#13014002. We thank Lewis Gaffney for assisting with data collection.

\section{Conflict of interest statement}

The authors declare that there are no conflicts of interest to disclose.

\section{Bibliography}

An, K.N., Ueba, Y., Chao, E.Y., Cooney, W.P., Linscheid, R.L., 1983. Tendon excursion and moment arm of index finger muscles. Journal of Biomechanics 16, 419-425. 
Boon, A.J., Oney-Marlow, T.M., Murthy, N.S., Harper, C.M., McNamara, T.R., Smith, J., 2011. Accuracy of electromyography needle placement in cadavers: non-guided vs. ultrasound guided. Muscle \& Nerve 44, 45-49.

Buchanan, T.S., Lloyd, D.G., Manal, K., Besier, T.F., 2004. Neuromusculoskeletal Modeling: Estimation of Muscle Forces and Joint Moments and Movements From Measurements of Neural Command. Journal of Applied Biomechanics 20, 367-395.

Chadwick, E.K., Blana, D., van den Bogert, A.J., Kirsch, R.F., 2009. A real-time, 3-D musculoskeletal model for dynamic simulation of arm movements. IEEE Transactions on Biomedical Engineering 56, 941-948.

Delp, S.L., Anderson, F.C., Arnold, A.S., Loan, P., Habib, A., John, C.T., Guendelman, E., Thelen, D.G., 2007. OpenSim: Open-Source Software to Create and Analyze Dynamic Simulations of Movement. IEEE Transactions on Biomedical Engineering 54, 1940-1950.

Delp, S.L., Grierson, A.E., Buchanan, T.S., 1996. Maximum isometric moments generated by the wrist muscles in flexion-extension and radial-ulnar deviation. Journal of Biomechanics 29, 1371-1375. 
Delp, S.L., Loan, J.P., Hoy, M.G., Zajac, F.E., Topp, E.L., Rosen, J.M., 1990. An interactive graphics-based model of the lower extremity to study orthopaedic surgical procedures. IEEE Transactions on Biomedical Engineering 37, 757-767.

Eilenberg, M.F., Geyer, H., Herr, H., 2010. Control of a powered ankle-foot prosthesis based on a neuromuscular model. IEEE Transactions on Neural Systems and Rehabilitation Engineering 18, 164-173.

Gonzalez, R.V., Buchanan, T.S., Delp, S.L., 1997. How muscle architecture and moment arms affect wrist flexion-extension moments. Journal of Biomechanics 30, 705712.

Higginson, J.S., Neptune, R.R., Anderson, F.C., 2005. Simulated parallel annealing within a neighborhood for optimization of biomechanical systems. Journal of Biomechanics 38, 1938-1942.

Higginson, J.S., Zajac, F.E., Neptune, R.R., Kautz, S.A., Delp, S.L., 2006. Muscle contributions to support during gait in an individual with post-stroke hemiparesis. Journal of Biomechanics 39, 1769-1777.

Hinkle, D., Wiersma, W., Jurs, S., 2003. Applied Statistics for the Behavioral Sciences, 5 ed. Houghton Mifflin, Boston. 
Holzbaur, K.R., Murray, W.M., Delp, S.L., 2005a. A model of the upper extremity for simulating musculoskeletal surgery and analyzing neuromuscular control. Annals of Biomedical Engineering 33, 829-840.

Holzbaur, K.R.S., Murray, W.M., Delp, S.L., 2005b. A Model of the Upper Extremity for Simulating Musculoskeletal Surgery and Analyzing Neuromuscular Control. Annals of Biomedical Engineering 33, 829-840.

Holzbaur, K.R.S., Murray, W.M., Gold, G.E., Delp, S.L., 2007. Upper limb muscle volumes in adult subjects. Journal of Biomechanics 40, 742-749.

Huang, H., Zhou, P., Li, G., Kuiken, T., 2009. Spatial filtering improves EMG classification accuracy following targeted muscle reinnervation. Annals of Biomedical Engineering 37, 1849-1857.

Jacobson, M.D., Raab, R., Fazeli, B.M., Abrams, R.A., Botte, M.J., Lieber, R.L., 1992. Architectural design of the human intrinsic hand muscles. Journal of Hand Surgery 17, 804-809.

Kamavuako, E.N., Farina, D., Yoshida, K., Jensen, W., 2012. Estimation of grasping force from features of intramuscular EMG signals with mirrored bilateral training. Annals of Biomedical Engineering 40, 648-656. 
Keir, P.J., Wells, R.P., Ranney, D.A., 1996. Passive properties of the forearm musculature with reference to hand and finger postures. Clinical Biomechanics (Bristol, Avon) 11, 401-409.

Knutson, J.S., Kilgore, K.L., Mansour, J.M., Crago, P.E., 2000. Intrinsic and extrinsic contributions to the passive moment at the metacarpophalangeal joint. Journal of Biomechanics 33, 1675-1681.

Kuiken, T.A., Li, G., Lock, B.A., Lipschutz, R.D., Miller, L.A., Stubblefield, K.A., Englehart, K.B., 2009. Targeted muscle reinnervation for real-time myoelectric control of multifunction artificial arms. Journal of the American Medical Association 301, 619-628.

Lehman, S.L., Calhoun, B.M., 1990. An identified model for human wrist movements. Experimental Brain Research 81, 199-208.

Li, G., Schultz, A.E., Kuiken, T.A., 2010. Quantifying pattern recognition-based myoelectric control of multifunctional transradial prostheses. IEEE Transactions on Neural Systems and Rehabilitation Engineering 18, 185-192.

Lieber, R.L., Jacobson, M.D., Fazeli, B.M., Abrams, R.A., Botte, M.J., 1992. Architecture of selected muscles of the arm and forearm: Anatomy and implications for tendon transfer. Journal of Hand Surgery 17, 787-798. 
Lloyd, D.G., Besier, T.F., 2003. An EMG-driven musculoskeletal model to estimate muscle forces and knee joint moments in vivo. Journal of Biomechanics 36, 765-776.

Lloyd, D.G., Buchanan, T.S., 1996. A model of load sharing between muscles and soft tissues at the human knee during static tasks. Journal of Biomechanical Engineering $118,367-376$.

Manal, K., Buchanan, T.S., 2013. An electromyogram-driven musculoskeletal model of the knee to predict in vivo joint contact forces during normal and novel gait patterns. Journal of Biomechanical Engineering 135, 021014.

Manal, K., Gonzalez, R.V., Lloyd, D.G., Buchanan, T.S., 2002. A real-time EMG-driven virtual arm. Computers in Biology and Medicine 32, 25-36.

Mathiowetz, V., Kashman, N., Volland, G., Weber, K., Dowe, M., Rogers, S., 1985. Grip and pinch strength: normative data for adults. Archives of Physical Medicine and Rehabilitation 66, 69-74.

Mercier, C., Reilly, K.T., Vargas, C.D., Aballea, A., Sirigu, A., 2006. Mapping phantom movement representations in the motor cortex of amputees. Brain 129, 2202-2210.

Messier, S.P., Legault, C., Loeser, R.F., Van Arsdale, S.J., Davis, C., Ettinger, W.H., DeVita, P., 2011. Does high weight loss in older adults with knee osteoarthritis affect 
bone-on-bone joint loads and muscle forces during walking? Osteoarthritis Cartilage 19, 272-280.

Muceli, S., Farina, D., 2012. Simultaneous and proportional estimation of hand kinematics from EMG during mirrored movements at multiple degrees-of-freedom. IEEE Transactions on Neural Systems and Rehabilitation Engineering 20, 371-378.

Murray, W.M., Delp, S.L., Buchanan, T.S., 1995. Variation of Muscle Moment Arms with Elbow and Forearm Position. Journal of Biomechanics 28, 513-525.

Neptune, R.R., Kautz, S.A., Zajac, F.E., 2001. Contributions of the individual ankle plantar flexors to support, forward progression and swing initiation during walking. Journal of Biomechanics 34, 1387-1398.

Norkin, C.C., White, D.J., 2009. Measurement of joint motion : a guide to goniometry, 4th ed. F.A. Davis, Philadelphia.

Perotto, A.O., 2005. Anatomical guide for the electromyographer: the limbs and trunk, 4th ed. Charles C. Thomas Publisher, Ltd., Springfield, IL.

Ramachandran, V.S., Hirstein, W., 1998. The perception of phantom limbs. The D. O. Hebb lecture. Brain 121 ( Pt 9), 1603-1630. 
Reddy, N.P., Gupta, V., 2007. Toward direct biocontrol using surface EMG signals: control of finger and wrist joint models. Medical Engineering \& Physics 29, 398-403.

Riek, S., Carson, R.G., Wright, A., 2000. A new technique for the selective recording of extensor carpi radialis longus and brevis EMG. Journal of Electromyography and Kinesiology 10, 249-253.

Saul, K.R., Murray, W.M., Hentz, V.R., Delp, S.L., 2003. Biomechanics of the Steindler Flexorplasty Surgery: A Computer Simulation Study. Journal of Hand Surgery 28A, 979986.

Shelburne, K.B., Pandy, M.G., 1998. Determinants of cruciate-ligament loading during rehabilitation exercises. Clinical Biomechanics 13, 403-413.

Shin, D., Kim, J., Koike, Y., 2009. A myokinetic arm model for estimating joint torque and stiffness from EMG signals during maintained posture. Journal of Neurophysiology $101,387-401$.

Tan, D.W., Schiefer, M.A., Keith, M.W., Anderson, J.R., Tyler, J., Tyler, D.J., 2014. A neural interface provides long-term stable natural touch perception. Science Translational Medicine 6, 257ra138. 
Weir, R.F., Troyk, P.R., DeMichele, G.A., Kerns, D.A., Schorsch, J.F., Maas, H., 2009. Implantable myoelectric sensors (IMESs) for intramuscular electromyogram recording. IEEE Transactions on Biomedical Engineering 56, 159-171.

Winters, J.M., 1990. Hill-Based Muscle Models: A Systems Engineering Perspective, in: Winters, J.M., Woo, S.L.-Y. (Eds.), Multiple Muscle Systems. Springer-Verlag, New York, pp. 69-93.

Wolpert, D.M., Flanagan, J.R., 2001. Motor prediction. Current Biology 11, R729-R732.

Zajac, F.E., 1989. Muscle and tendon: properties, models, scaling, and application to biomechanics and motor control. Critical Reviews in Biomedical Engineering 17, 359411.

Zajac, F.E., Neptune, R.R., Kautz, S.A., 2002. Biomechanics and muscle coordination of human walking. Part I: introduction to concepts, power transfer, dynamics and simulations. Gait \& Posture 16, 215-232.

Zhang, X., Huang, H., 2015. A real-time, practical sensor fault-tolerant module for robust EMG pattern recognition. Journal of Neuroengineering and Rehabilitation 12, 18. 


\section{Tables}

Table 1: Musculoskeletal parameter constraints during optimization.

\section{Parameter (units)}

optimal contractile element length $(m)$

maximum isometric contractile element force $(\mathrm{N})$

moment arm, wrist $(\mathrm{m})$

moment arm, $\mathrm{MCP}^{*}(\mathrm{~m})$

contractile element length in neutral posture (unitless)

passive elastic element stiffness $\left(\mathrm{N} / \mathrm{m}^{2}\right)$

${ }^{*} m a_{M C P}=0$ for wrist flexor/extensor muscles

\author{
Constraint \\ $0.01 \leq L_{0}^{C E} \leq 0.5$ \\ $10 \leq F_{n}^{C E} \leq 1000$ \\ $0.001 \leq m a_{\text {wrist }} \leq 0.05$ \\ $0.001 \leq m a_{M C P} \leq 0.05$ \\ $0.75 L_{0}^{C E} \leq L_{\theta=0}^{C E} \leq 1.25 L_{0}^{C E}$ \\ $10 \leq K^{P E E} \leq 200$
}


Table 2: Optimization algorithm parameters defined for Matlab's GlobalSearch function and simulated annealing.

\begin{tabular}{clcllc}
\hline \multicolumn{1}{c}{ GlobalSearch } & & \multicolumn{2}{c}{ simulated annealing } \\
fmincon & "Maxlter" & 8000 & cooling rate (\%) & 10 \\
solver & "MaxFunEvals" & 8000 & number of cooling cycles & 20 \\
$\begin{array}{c}\text { settings } \\
\text { GlobalSerch }\end{array}$ & "TolFun" & 0.01 & number of searches in each cycle & 10000 \\
settings & "NumTrialPoints" & 700 & & \\
\hline
\end{tabular}


Table 3: Optimized musculoskeletal parameters for the four virtual muscles for all subjects $\left(L_{0}^{C E}=\right.$ optimal muscle length $(\mathrm{m}), F_{0}^{C E}=$ maximum isometric muscle force $(\mathrm{N}), m a$ $=$ moment $\operatorname{arm}(\mathrm{m}), L_{\theta=0}^{C E}=$ muscle length in neutral posture $(\mathrm{m}), K^{P E E}=$ parallel elastic element stiffness $\left.\left(\mathrm{N} / \mathrm{m}^{2}\right)\right)$.

\begin{tabular}{|c|c|c|c|c|c|}
\hline \multirow{2}{*}{ subject } & \multirow[b]{2}{*}{ parameter } & \multicolumn{4}{|c|}{ virtual muscle } \\
\hline & & wrist extensor & MCP extensor & wrist flexor & MCP flexor \\
\hline \multirow{6}{*}{$\mathrm{AB} 1$} & $L_{0}^{C E}$ & 0.238 & 0.054 & 0.109 & 0.179 \\
\hline & $F_{0}^{C E}$ & 1000 & 42 & 234 & 257 \\
\hline & $m a_{\text {wrist }}$ & -0.050 & -0.013 & 0.050 & 0.050 \\
\hline & $m a_{M C P}$ & 0 & -0.050 & 0 & 0.050 \\
\hline & $L_{\theta=0}^{C E}$ & 1.109 & 0.750 & 1.153 & 1.122 \\
\hline & $K^{P E E}$ & 53 & 22 & 160 & 54 \\
\hline \multirow{6}{*}{$A B 2$} & $L_{0}^{C E}$ & 0.257 & 0.071 & 0.133 & 0.026 \\
\hline & $F_{0}^{C E}$ & 332 & 59 & 530 & 265 \\
\hline & $m a_{\text {wrist }}$ & -0.049 & -0.029 & 0.041 & 0.004 \\
\hline & $m a_{M C P}$ & 0 & -0.034 & 0 & 0.018 \\
\hline & $L_{\theta=0}^{C E}$ & 1.130 & 1.033 & 1.046 & 1.250 \\
\hline & $K^{P E E}$ & 149 & 25 & 182 & 67 \\
\hline \multirow{6}{*}{ AB3 } & $L_{0}^{C E}$ & 0.150 & 0.171 & 0.412 & 0.146 \\
\hline & $F_{0}^{C E}$ & 366 & 80 & 651 & 316 \\
\hline & $m a_{\text {wrist }}$ & -0.050 & -0.028 & 0.050 & 0.038 \\
\hline & $m a_{M C P}$ & 0 & -0.050 & 0 & 0.050 \\
\hline & $L_{\theta=0}^{C E}$ & 1.225 & 1.016 & 1.197 & 1.130 \\
\hline & $K^{P E E}$ & 200 & 200 & 200 & 75 \\
\hline \multirow{6}{*}{$\mathrm{AB} 4$} & $L_{0}^{C E}$ & 0.239 & 0.039 & 0.250 & 0.442 \\
\hline & $F_{0}^{C E}$ & 584 & 130 & 261 & 99 \\
\hline & $m a_{\text {wrist }}$ & -0.050 & -0.019 & 0.049 & 0.025 \\
\hline & $m a_{M C P}$ & 0 & -0.038 & 0 & 0.049 \\
\hline & $L_{\theta=0}^{C E}$ & 1.032 & 0.762 & 1.023 & 1.161 \\
\hline & $K^{P E E}$ & 200 & 10 & 194 & 196 \\
\hline \multirow{6}{*}{ AB5 } & $L_{0}^{C E}$ & 0.022 & 0.012 & 0.500 & 0.047 \\
\hline & $F_{0}^{C E}$ & 1000 & 272 & 906 & 108 \\
\hline & $m a_{\text {wrist }}$ & -0.015 & -0.005 & 0.013 & 0.017 \\
\hline & $m a_{M C P}$ & 0 & -0.012 & 0 & 0.032 \\
\hline & $L_{\theta=0}^{C E}$ & 1.182 & 1.241 & 1.208 & 1.250 \\
\hline & $K^{P E E}$ & 149 & 10 & 136 & 101 \\
\hline \multirow{6}{*}{ TRA } & $L_{0}^{C E}$ & 0.078 & 0.019 & 0.070 & 0.183 \\
\hline & $F_{0}^{C E}$ & 256 & 42 & 107 & 56 \\
\hline & $m a_{\text {wrist }}$ & -0.029 & -0.001 & 0.031 & 0.050 \\
\hline & $m a_{M C P}$ & 0 & -0.015 & 0 & 0.016 \\
\hline & $L_{\theta=0}^{C E}$ & 1.125 & 0.776 & 0.857 & 0.968 \\
\hline & $K^{P E E}$ & 10 & 118 & 97 & 200 \\
\hline
\end{tabular}




\section{Figure Captions}

Figure 1: EMG electrode and reflective marker placement for EMG and kinematic data recording, respectively. Illustration shows the anterior aspect of the forearm. Dotted circles indicate marker/electrode located on the anterior aspect of the forearm. $\mathrm{PIP}=$ proximal interphalangeal; $\mathrm{MCP}=$ metacarpophalangeal.

Figure 2: Measured and predicted wrist and MCP joint angles during (A) wrist-only, (B) MCP-only, and $(C)$ simultaneous wrist and MCP varying speed movements from subject AB5.

Figure 3: Measured and predicted wrist and MCP joint angles during (A) wrist-only, (B) MCP-only, and $(C)$ simultaneous wrist and MCP varying speed movements from subject TRA.

Figure 4: Pearson's correlation coefficient $(r)$ between measured and predicted joint angles during single-joint and simultaneous multi-joint (wirst and MCP) movements.

Figure 5: Mean model-predicted and experimentally-measured passive flexion, extension, and neutral joint moments at the wrist (top) and MCP (bottom) joints. Bars indicate $+/-1$ standard deviation.

Figure 6: Mean model-predicted and experimentally-measured maximum isometric joint moments at the wrist (neutral posture) and MCP ( $45^{\circ}$ flexion) joints. MCP extension moments were excluded because experimental data were not available. *Experimental wrist data adapted from (Delp et al., 1996), and MCP data adapted from (Mathiowetz, et al. 1985). MCP joint moment was computed from linear force data from the right hand of 20-24 year old males assuming a $2 \mathrm{~cm}$ moment arm between the dynamometer handle and MCP joint. 
Figure 7: Correlation between measured and predicted joint angles during simultaneous wrist-MCP movement when musculoskeletal parameter values were computed either by Matlab's GlobalSearch function or simulated annealing $(n=10$ routines with each algorithm) for subject $A B 4$. 


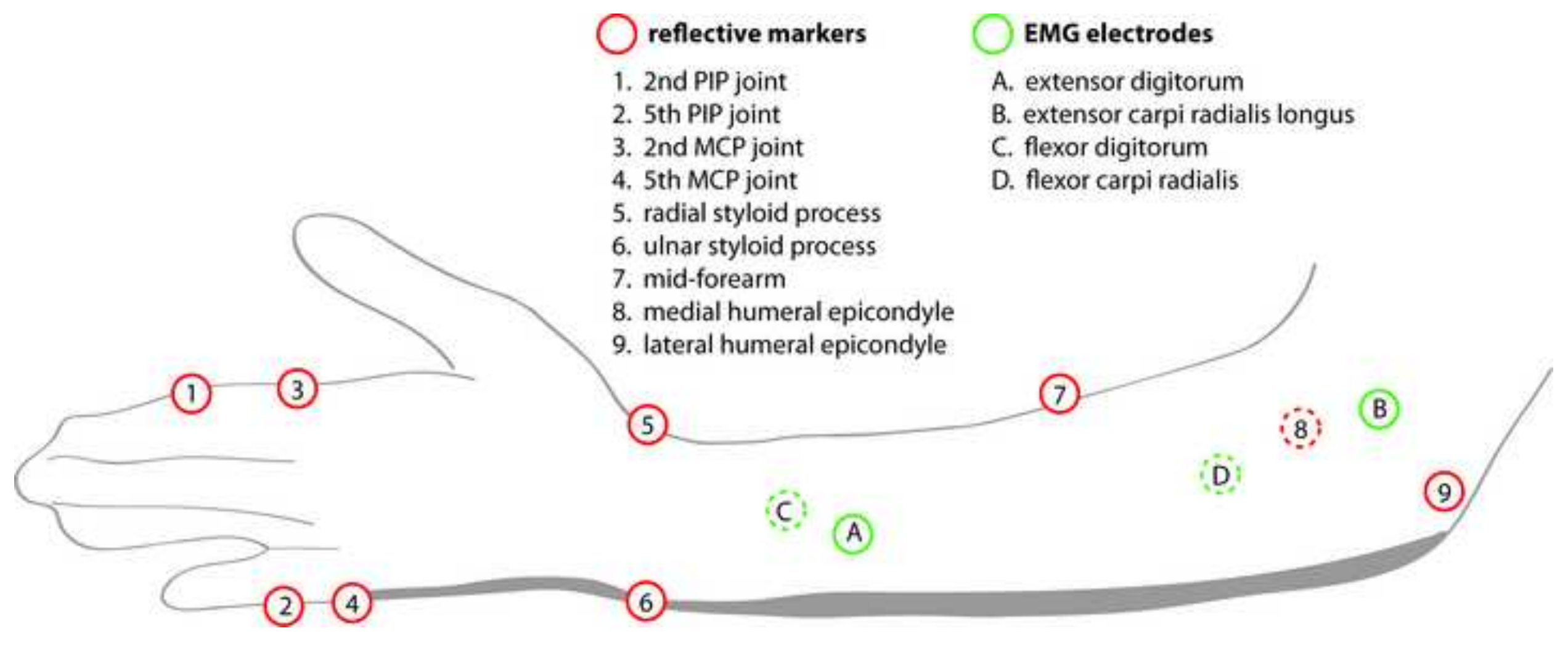


Figure 2
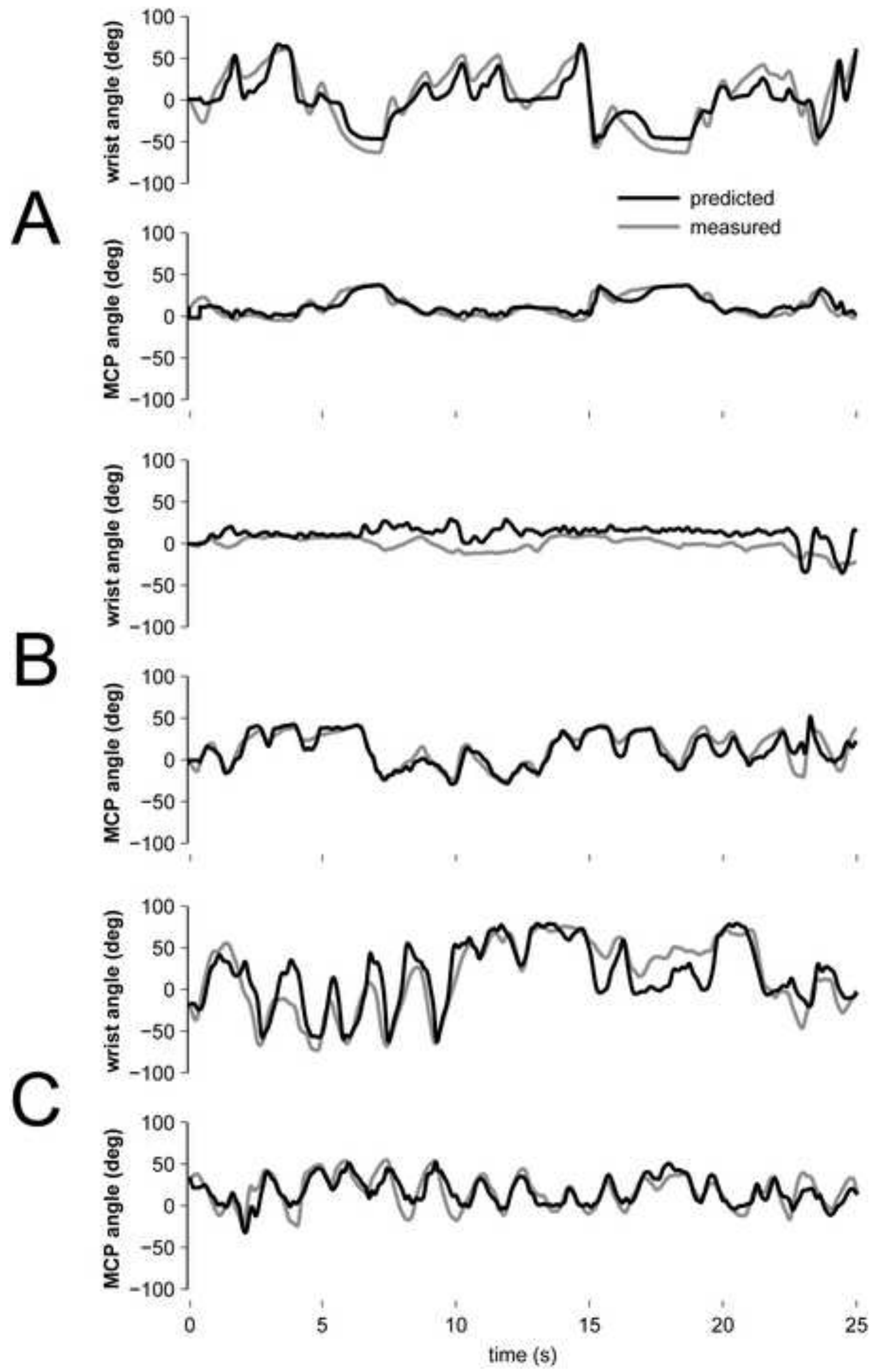
Figure 3
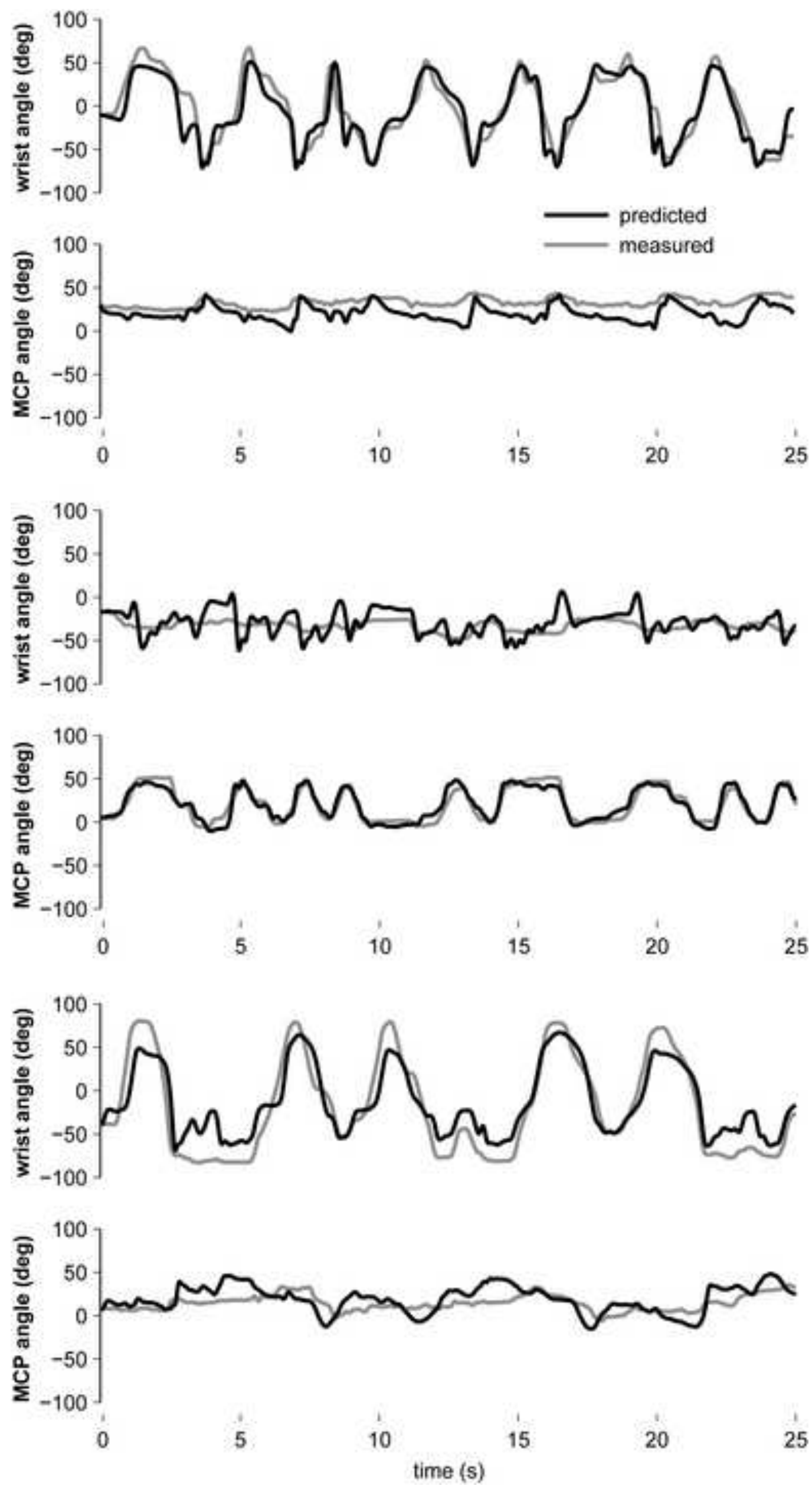


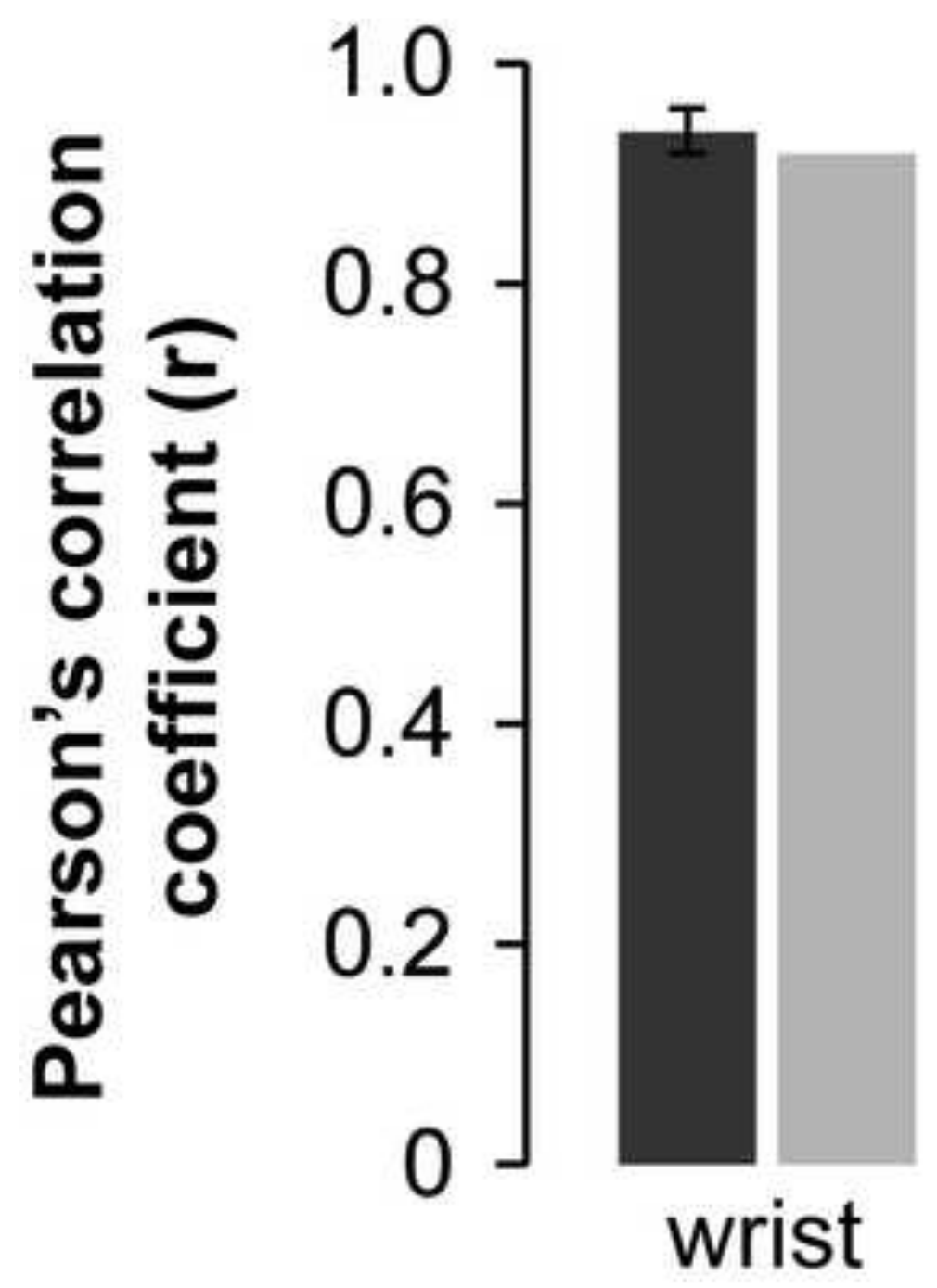

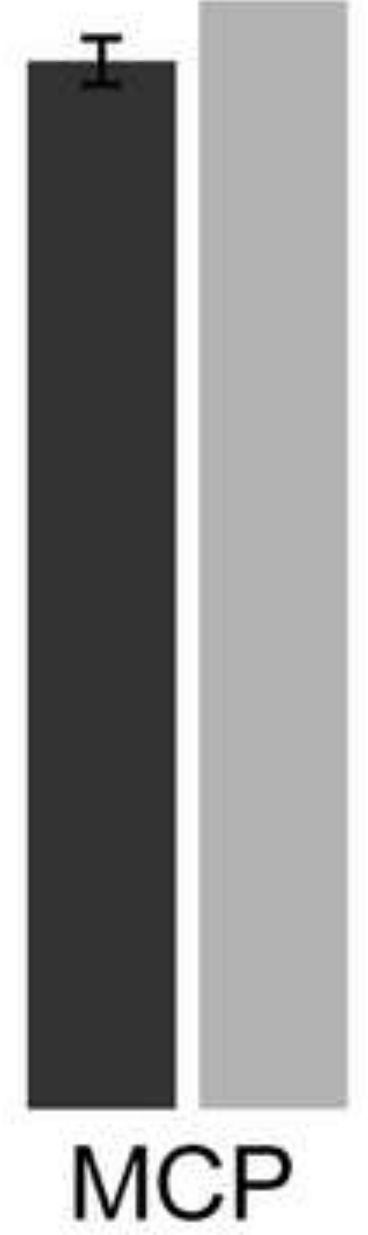

single joint
- $\mathrm{AB}$

TRA

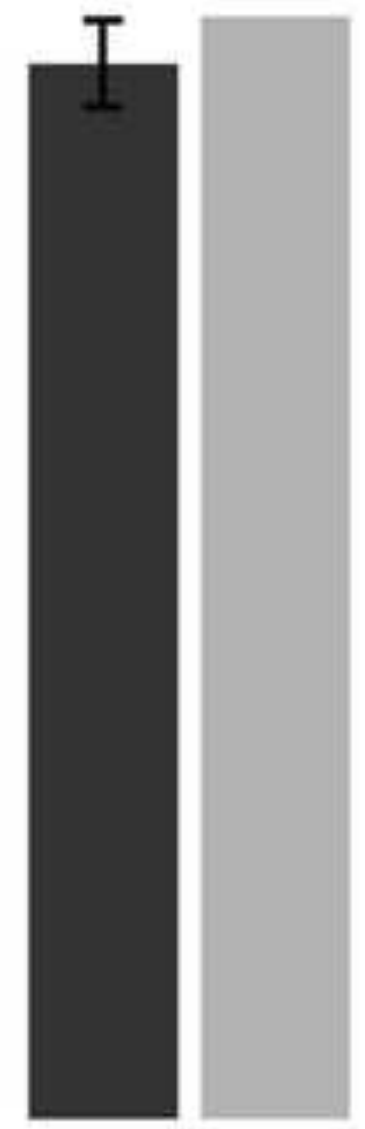

wrist

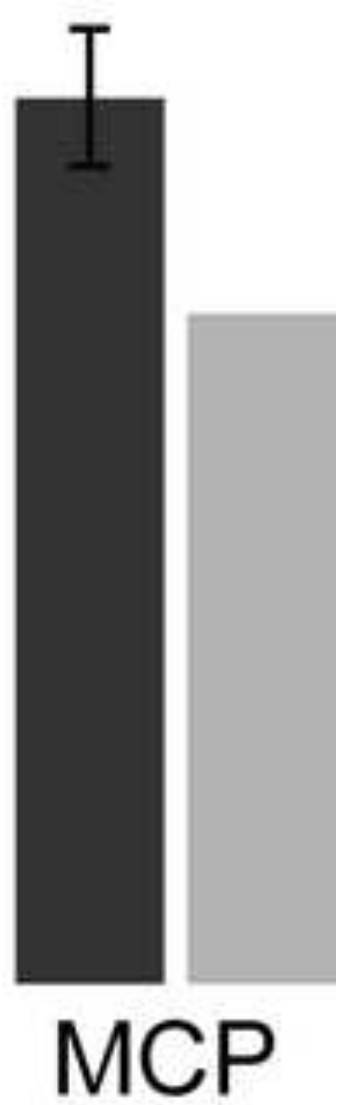

simultaneous wrist + MCP 
Figure 5
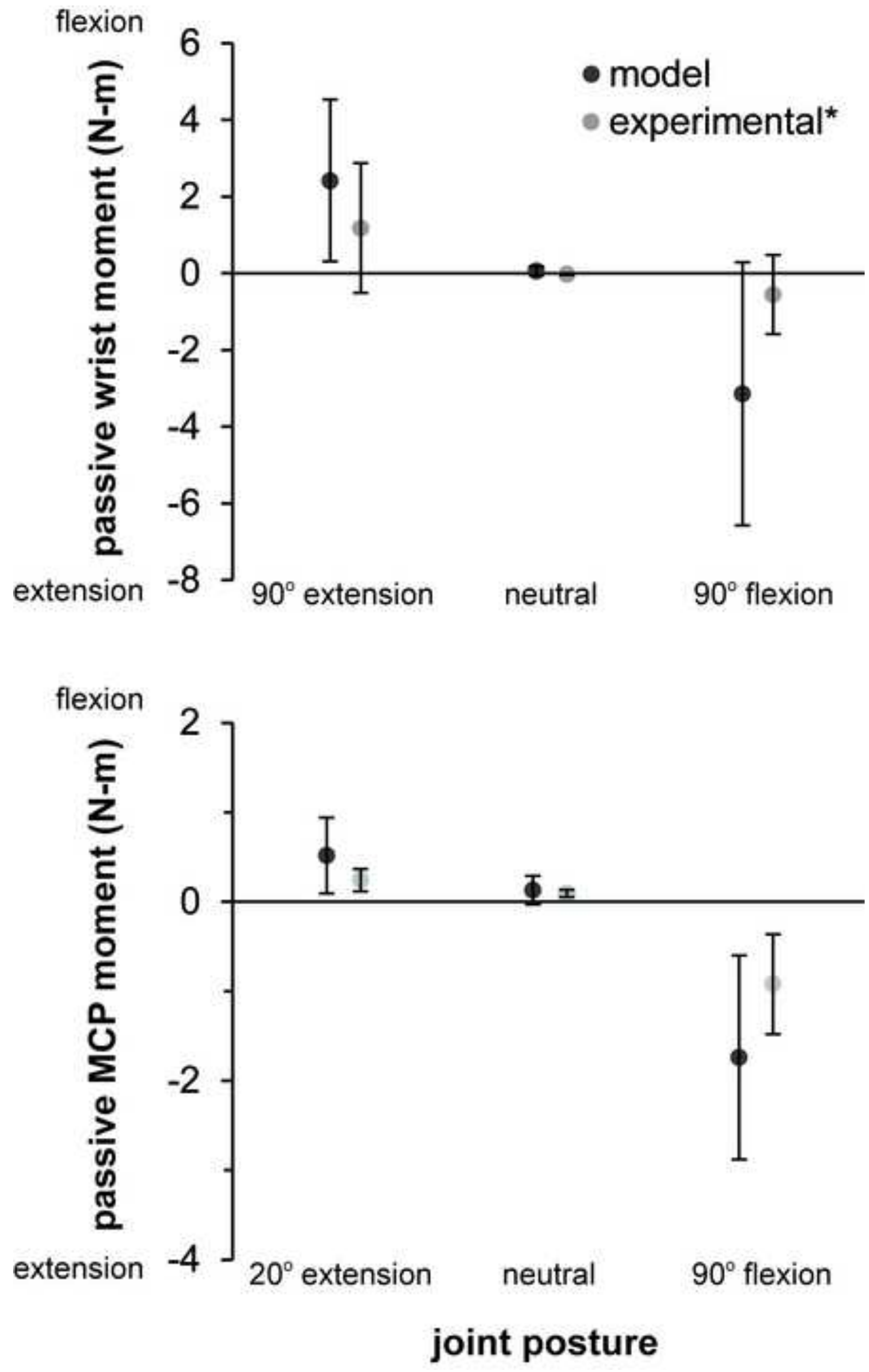


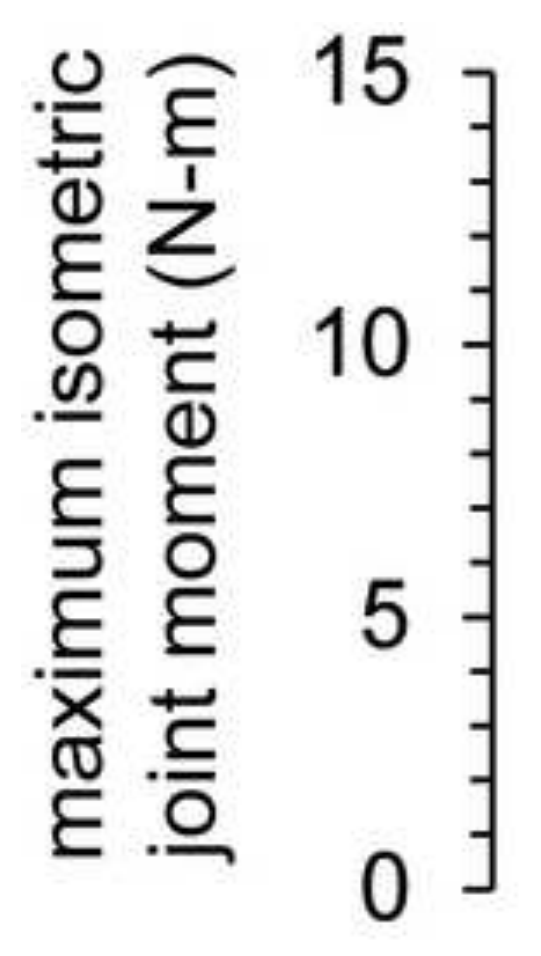

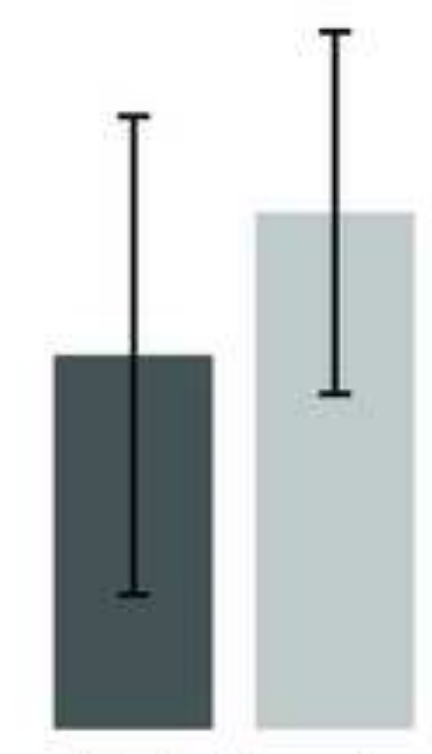

wrist flexion

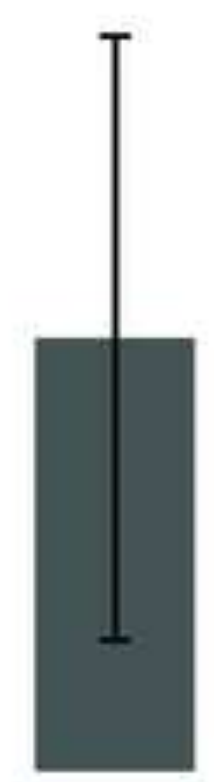

wrist extension model

- experimental ${ }^{*}$

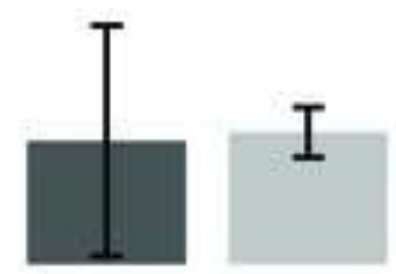

MCP flexion 

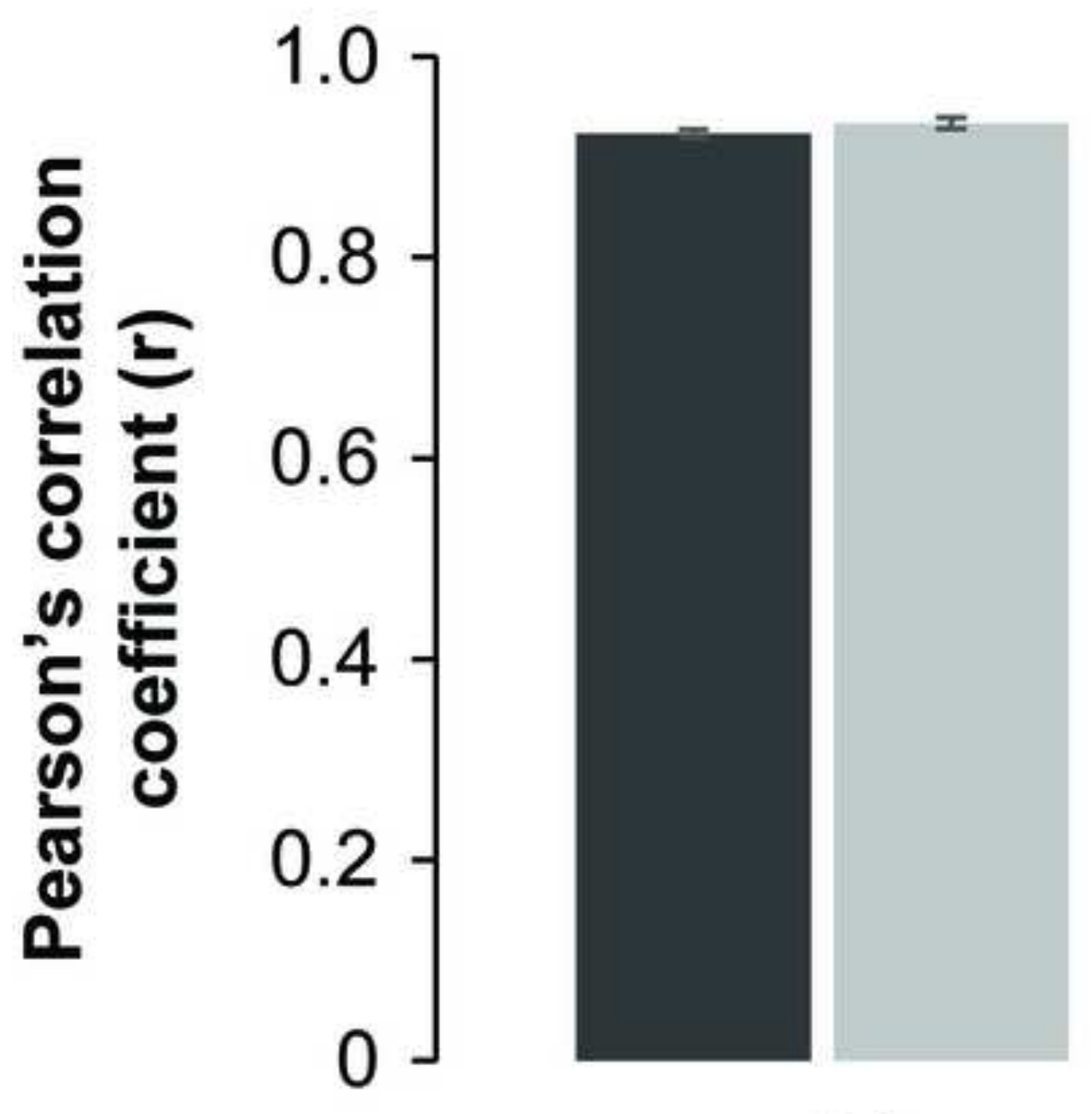

wrist

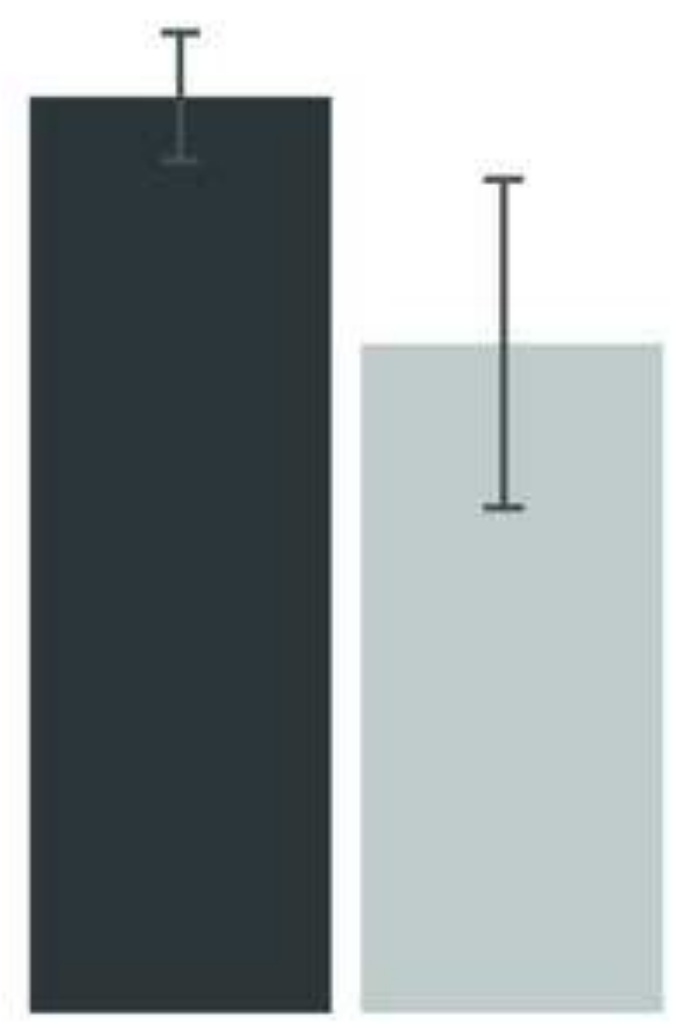

MCP 